\title{
La profanation du sacré dans certains romans de Saramago et de Kundera: approche à l'étude de l'ironie et la parodie dans le roman contemporain
}

\author{
Graciela ESTRADA VARGAS \\ Universidad Nacional Autónoma de México
}

\begin{abstract}
El artículo se ocupa de la novela contemporánea. A través del análisis de ciertas novelas de Saramago y Kundera publicadas entre 1970 y 1990, el presente estudio destaca la manera en que ambos novelistas utilizan la ironía y la parodia para cuestionar "la verdad" que pesa sobre el espíritu de las sociedades contemporáneas.

La parodia bíblica es un punto de convergencia entre los textos estudiados, aunque se distinguen por sus particularidades estilísticas. Nuestras principales fuentes teóricas son los distintos trabajos sobre parodia e ironía escritos por Linda Hutcheon.
\end{abstract}

PALABRAS CLAVE: Saramago, Kundera, novela contemporánea, ironía, parodia.

This article studies the contemporary novel. Focusing on some novels by Saramago and Kundera, published between the 1970's and the 1990's, this study highlights how both novelists, by using parody and irony, reappraise "the truth" that influences the spirit of contemporary societies.

The Bible parody is, despite stylistic particularities, a point of convergence in these writings. Our theoretical main sources are Hutcheon's works on parody and irony.

KEY WORDS: Saramago, Kundera, contemporary novel, parody, irony.

Ce travail propose une réflexion sur le roman contemporain, en analysant l'ironie et la parodie dans des oeuvres de Saramago et Kundera, notamment $O$ Evangelho segundo Jesus Cristo (1991) du Nobel portugais et La vie est ailleurs (1973, revue en 1985) de l'auteur franco-tchèque; toutefois certaines mentions à d'autres romans seront évoquées. ${ }^{1}$

Deux romanciers issus de cultures et langues différentes, mais qui vivent et écrivent à la même époque, ont-ils des traits communs? Quant aux notions d'ironie et de parodie, quelles sont leurs définitions et caractéristiques dans l'actualité?

${ }^{1}$ La valse aux adieux (1973), Levantado do Chão (1980), Memorial do Convento (1982), História do Cerco de Lisboa (1989) et Ensaio sobre a Cegueira (1995). 


\section{$136 \square$ LA PROFANATION DU SACRÉ DANS CERTAINS ROMANS}

Pour cette étude, nous tiendrons compte des différents ouvrages théoriques de Linda Hutcheon, de l'étude de Wayne Booth, A Rhetoric of Irony (1974) et de celle de Margaret Rose, Parody: ancient, modern, and post-modern (1993).

La parodie de la Bible dans Evangelho, le roman écrit par Saramago, est annoncée au lecteur dès le titre, par la reprise d'un élément biblique, celui de l'évangile. L'épigraphe du roman est une citation de l'Evangile de Lucas, 1,1-4, les noms des personnages (Jésus, Joseph, Tiago, Tomas, Lazare), certains de leurs attributs (le métier de pêcheur, Jésus "fils de Dieu", l'accomplissement des miracles) et le contexte (peuple hébreu sous domination romaine) sont autant d'éléments qui renvoient à l'intertexte biblique.

C'est cette grande ressemblance entre le roman de Saramago et l'intertexte biblique qui sert d'arrière plan au contraste ironique. Le lecteur, connaissant les événements et dogmes de la Bible, les retrouve dans le roman où il est confronté à la rupture de ses attentes.

Dans son article "Ironie, satire et parodie" (1981), Hutcheon parle de l'ironie comme d'un trope qui a une place importante dans les genres de la parodie et de la satire. Bien que l'acte et la forme parodiques sont ceux de l'incorporation, leur fonction étant de séparer et de créer contraste. ${ }^{2}$ La parodie moderne nécessite de la distance critique ironique (TP: 34), "distance critique entre le texte d'arrière-plan qui est parodié et le nouveau texte enchâssant" ("Ironie et": 468). La parodie qui utilise l'ironie comme principal moyen d'accentuer ou d'établir le contraste parodique, implique de prendre en compte le contexte énonciatif pour sa production et sa réception (TP: 34). La parodie moderne combine "l'hommage ${ }^{3}$ respectueux" et "le pied de nez ironique" ("Ironie et": 469).

Dans Evangelho, les actions de Jésus connaissent des variantes importantes, par exemple dans l'épisode de la résurrection de Lazare:

[...] só falta que Jesus [...] diga, Lázaro, levanta-te

[...] mas é neste instante [...] Maria de Magdala [...]

diz, Ninguém na vida teve tantos pecados que mereça

morrer duas vezes, então Jesus deixou cair os braços e saiu para chorar (Evangelho: 428) chapitre 23.

La parodie est présente car les personnages sont ceux du texte source: Jésus, Lazare et sa sœur. Pourtant une différence est intégrée: le miracle de la résurrection de Lazare n'est pas accompli. Dans les Evangiles bibliques Jésus rend la vie à son ami Lazare, alors que dans le roman de Saramago, Evangelho, Jésus, à cause de Maria de Magdala, n'achève pas ce miracle.

Dans l'introduction à Irony's Edge (1994), Hutcheon écrit que c'est l'ironie qui donne à la parodie sa dimension critique et sa marque de différence au cœur de la similarité.

\footnotetext{
${ }^{2}$ Ce contraste est déjà dans la racine grecque du terme ("face à"), signale Hutcheon dans $T P$.

${ }^{3}$ Hutcheon donne comme exemple ce que Jane Austen a fait dans Amour et Amitié un "hommage critique" envers les conventions du roman épistolaire ("Ironie et": 475).
} 
La parodie est une trans-contextualisation ironique et inversement, elle est répétition avec différence. Il y a une distance implicite entre le texte parodié et la nouvelle œuvre et cette distance est souvent signalée par l'ironie. Cette dernière peut être badine ou rabaissante, critique constructive comme destructrice (l'ironie a une nature transidéologique).

La parodie biblique dans Evangelho est présente aussi sous la forme de commentaires métafictionnels. Le narrateur mentionne avec clarté que la source du discours qu'il attribue à Joseph est le Livre de Job et explique la nouvelle signification qu'elle prend dans le contexte romanesque: "Disse José, Deus conhece todos os meus caminhos e conta todos os meus passos, e estas palavras do carpinteiro, que podemos encontrar no Livro de Job, significavam, no contexto da discussão, que [...] José reconhecia e protestava a sua obediência ao Senhor" (Evangelho: 58).

Dans un autre roman du Portugais, Ensaio, l'Évangile de Luc est parodié par la reprise du Bon Samaritain. Le narrateur compare le personnage qui aide le premier aveugle à cette figure biblique: "Não fiz mais que o meu dever, justificou o bom samaritano" (Ensaio: 15). La comparaison devient incongrue lorsque le faux samaritain du roman vole la voiture de l'aveugle: "O santinho do teu protector, a boa alma, levou-nos o carro" (Ensaio, 20) "afinal falso samaritano" (Ensaio: 25). C'est un voleur déguisé en Bon Samaritain. Kundera parodie lui aussi le Nouveau Testament. Le narrateur de La vie est ailleurs, en adopte le ton et le lexique: “Au commencement, il n'y avait que lui, Jaromil" (La vie est ailleurs: 436) qui renvoient à l'ouverture de l'Evangile de Jean: "Au commencement était le Verbe" (Jn 1,1). La parodie de la source biblique se poursuit dans le roman de Kundera, mais cette fois elle renvoie au Livre de la Genèse: "Ensuite Jaromil a créé Xavier, son double..." (La vie est ailleurs: 436). Deux éléments du registre biblique sont repris ici: le commencement et la création. Insérés dans un contexte nouveau et différent, ils permettent de donner un sens ironique à un événement raconté quelques pages plus loin:

Jaromil s'approcha de la fenêtre et il vit le divan [...] il vit deux corps nus qui s'étreignaient $[\ldots]$ le corps de la femme recouvert par le corps de l'homme appartenait à la cinéaste [...] Mais qui est cet homme? Mon Dieu! [...] Xavier avec une femme! [...] pourtant Xavier et Jaromil devaient être une seule et même personne! Comment se peut-il que Xavier le trahisse? Mon Dieu, comment se peut-il qu'il fasse l'amour sous ses yeux avec son amie? (La vie est ailleurs: 454).

Jaromil est une sorte d'alter ego divin car, comme Dieu, il est créateur de Xavier. Jaromil voit la nudité de sa créature et de la femme qu'il désire, la cinéaste. D'ailleurs, Xavier n'est pas seulement décrit comme le double de Jaromil (La vie est ailleurs: 436), en effet ils forment tous deux "une seule et même personne", union hyperbolique qui renvoie au dogme catholique de la Trinité où plusieurs personnes ne font qu'une. Les parallélismes entre Jaromil et Dieu (les créateurs), la cinéaste et Eve (la femme nue/ objet du désir) et finalement entre Xavier et Adam (les créatures), font de cette scène de La vie est ailleurs une parodie biblique ironique. Or, dans l'intertexte biblique de la 
Genèse, Dieu chasse l'homme et la femme du paradis, nus et pleins de honte, alors que dans le roman kunderien, Jaromil, le créateur (poète) est celui qui quitte le lieu de la scène, seul et ridiculisé: "Il ne pouvait pas supporter de rester ici plus longtemps [...] il ne voulait rien voir, il ne regardait ni à gauche ni à droite, et il traversa la chambre d'un pas rapide $[\ldots]$ Il sortit dans l'entrée [...] prit son chapeau et sortit en courant de la villa" ( $L a$ vie est ailleurs: 455 ).

Cette scène constitue une parodie ironique de l'expulsion d'Eve et Adam du paradis car les trois personnages renvoient à la source biblique. Elle est ironique car les rôles sont inversés: le Créateur (Jaromil/Dieu) abandonne la scène tandis que restent l'homme et la femme. L'inversion de l'ordre biblique d'un événement qui sert d'explication, voire de cause métaphysique, au péché originel de l'humanité, banalise l'importance de cette souillure. En même temps, la conduite impuissante de Jaromil, incongrue si on la compare à celle de Dieu dans la Bible, rend le personnage risible.

La transposition d'éléments de l'intertexte biblique dans le nouveau contexte du roman sert à la composition d'un scénario qui renverse de manière inattendue et moqueuse le sens du pré-texte. Le lecteur ne peut pas s'empêcher de participer à la ridiculisation du personnage, il y est invité par le ton moqueur du narrateur, signalé par l'utilisation des expressions pieuses et des signes d'admiration: "Mon Dieu!", "cette scène-là!", "une femme!", "une seule et même personne!", "Mon Dieu" (La vie est ailleurs: 454) et parce que la scène est précédée d'une autre où le personnage, Jaromil, est soulevé et transporté alors qu'il "se débattait comme un poisson tendre et désespéré" (La vie est ailleurs: 448). La parodie biblique place la charge mythique du texte source dans une autre situation. Placée dans un tout autre contexte elle acquiert des significations nouvelles et s'établit ainsi une relation de différence par rapport au texte parodié, donnant lieu à l'ironie et au rire.

Dans le même roman, lorsque Jaromil est vu avec une femme laide par un camarade moqueur, un commentaire du narrateur compare la réaction de Jaromil à celle que les Evangiles (de Jean et Marc) attribuent à l'apôtre Pierre: "Il renia son amie comme Pierre renia le Christ [...] il en parlait avec dédain. Mais de même que Pierre resta fidèle au Christ, Jaromil, dans son for intérieur, resta fidèle à son amie" (La vie est ailleurs: 314). Kundera transpose le passage sombre de la trahison de l'apôtre dans une scène où s'illustre la lâcheté adolescente de Jaromil provoquée par un fait trivial: la laideur de son amie. L'effet d'incongruité est créé par l'écart entre la nature des faits mis en rapport: l'apôtre renie Dieu, Jaromil renie sa petite amie. La comparaison, en même temps qu'elle hyperbolise le dédain de Jaromil, banalise celui de l'apôtre.

La parodie ironique faite par Kundera travaille dans deux directions, elle accentue et détruit.

Dans Irony's edge, la théoricienne cite le romancier franco-tchèque, qui voit dans l'ironie la faculté de nier les certitudes et de montrer l'ambiguïté du monde ( $L a$ vie est ailleurs: 15). Selon Hutcheon, l'ironie n'irrite pas seulement au niveau intellectuel mais aussi sur un plan émotionnel. Elle a une charge affective (Booth: 44). C'est ce qu'elle appelle le 'tranchant' ("edge") ou l' 'aiguillon' ("sting") de l'ironie et c'est 
ce qu'elle souligne dans le titre-même de son étude, Irony's edge. Dans l'exemple cité auparavant, l'ambiguïté que la parodie ironique contient, est le terreau du tranchant ironique signalé dans la théorie de Hutcheon.

\section{L'ironie de la divinité perfectible}

Le discours religieux dans les romans du corpus fait l'objet d'une mise en question systématique des qualités de ses acteurs (saints, prêtres, anges, divinités) par des réflexions sur leurs actions et objets (invraisemblance des miracles, inauthenticité des reliques).

La méditation sur l'invraisemblance des miracles est une source d'ironie chez Saramago. L'attribution de conduites blâmables aux représentants religieux (hypocrisie, vices) comme dans le cas du prêtre de Levantado do Chão, se retrouve aussi chez Kundera, qui donne à ses personnages les qualités de "prédicateurs" et d' "anges" (Bertlef, La valse aux adieux) tout en leur prêtant des conduites très décalées par rapport à leur désignation, créant de l'incongruité. Pour sa part, Saramago utilise des registres inattendus, voire inappropriés, dans le traitement du sacré (reliques, prières, actions miraculeuses). Le discours religieux et ses valeurs dogmatiques sont repris depuis une perspective nouvelle dans les romans saramaguiens. Le lecteur se trouve placé là où la vieille perspective usée, imposée et apparemment invariable perd sa valeur d'irrévocable.

Dans Evangelho, l'évocation de l'un des miracles attribués à Jésus, celui d'avoir libéré un homme possédé par les démons, parodie les Evangiles bibliques (Matthieu, Marc, Luc). Le roman de Saramago semble de prime abord reprendre l'image du Jésus des textes bibliques, celle d'un personnage qui interpelle les démons avec autorité, comme le montre l'utilisation de l'adverbe 'impérieusement' et l'adjectif 'immonde': "Disse Jesus, imperiosamente, Sai desse homem, espírito imundo..." (Evangelho: 354355, chapitre 21). Mais dans la suite du dialogue, l'autorité de Jésus est sapée par le ton conciliant qu'il utilise pour s'adresser aux démons: 'où voulez-vous aller?', lesquels finissent par choisir eux-mêmes leurs prochaines victimes: "Perguntou Jesus, E para onde querem vocês ir [...] Manda-nos para os porcos e entraremos neles. Jesus pensou e pareceu-lhe que era uma boa solução..." (Evangelho: 355).

Le dialogue, rapporté par le narrateur, montre que Jésus approuve la décision prise par les démons. Suivent les commentaires du narrateur rapportant les conséquences néfastes de l'action: les cochons possédés se précipitent et tombent dans la mer où ils meurent noyés, libérant ainsi les démons (Evangelho: 355). L'imprévoyance de Jésus est soulignée par la répétition de la phrase "não lhe ocurreu [a Jesus]" / 'il n'y a pas pensé' et l'adverbe "desgraçadamente" / 'malheureusement': "A idea de que [...] poderiam os gentios ingerir também os demónios [...] não ocurreu a Jesus, como também não lhe ocurreu o que depois desgraçadamente aconteceu..." (Evangelho: 355$)$.

Dans la suite du commentaire, le narrateur semble prendre parti pour sa cible et vouloir justifier le manque de prévoyance du personnage, ce qui renforce l'ironie: 
"mas a verdade é que nem mesmo um filho de Deus, aliás ainda não habituado a tão alto parentesco, poderia prever [...] todas as consequências dum simples lance, duma decisão simples" (Evangelho: 355 ).

Le choix de désigner Jésus par l'article indéfini ' $u n$ ' fils au lieu de 'le' fils de Dieu place le personnage au niveau de l'humanité commune, ce qui transgresse ou contredit le dogme catholique: Jésus est ' un' parmi des autres. La répétition du mot 'simple' pour évoquer la légèreté avec laquelle Jésus décide, accentue de manière ironique l'incapacité du personnage. L'ironie réside, en conséquence, dans le fait que le narrateur feint de prendre parti pour Jésus et dans la transgression du dogme.

Pourtant, Jésus n'est pas présenté comme naïf car, même s'il est incapable de prévoir les erreurs, il est capable de les mesurer lorsqu'il les a commises. Son discours témoigne de cette conscience: "Escuta-me, ó Senhor, ou tu escolheste mal o filho que disseram que eu sou e há-de cumprir os teus desígnios, ou entre os teus mil poderes falta o duma inteligência capaz de vencer a do diabo..." (Evangelho: 357).

Donc le but n'est pas de ridiculiser le personnage de Jésus, mais de le faire choir de son haut rang de divinité pour l'inscrire du côté de l'humanité, où l'erreur et l'incapacité à prévoir les conséquences futures sont possibles et normales. De plus, dans son discours, Jésus interpelle Dieu par 'Seigneur' non pas par 'Mon père', manifestant ainsi sa conscience que ce sont les autres qui lui attribuent le statut de 'fils de Dieu'.

Par la technique de la répétition le narrateur rappelle que les premiers à avoir reconnu Jésus comme "fils de Dieu" furent les démons, ceux qui ont provoqué la mort des cochons. Trois fois au cours du XXI ${ }^{\text {ème }}$ chapitre le narrateur répète cette idée:

[...] esta foi a primeira vez que em público [...] uma voz se levantou, e voz diabólica ela era, para anunciar que este Jesus de Nazaré era filho de Deus, o que ele próprio até aí ignorava (Evangelho: 354).

[...] a insólita revelação, feita pelos demónios de que Jesus era filho de Deus... (Evangelho: 356).

[...] a tremendíssima questão de ser Jesus, segundo a revelação dos demónios, filho de Deus (Evangelho: 357).

Les commentaires du narrateur à propos du sort des cochons (morts suite à leur possession par les démons lesquels ont ensuite réussi à se libérer) offrent de nouvelles perspectives au lecteur par rapport au texte biblique. Les trois Evangiles qui racontent ce miracle (Mc 5, 1, Mt 8, 28, Lc 8, 26) mentionnent la mort des cochons et suggèrent l'ennui de leurs propriétaires, mais ne réfléchissent pas aux autres conséquences. Les commentaires du narrateur remettent en question la décision de Jésus et montrent des victimes innocentes (autant les cochons que leurs propriétaires qui ont vu périr leur moyen de subsistance).

La phrase mentionnant que l'action miraculeuse a fait le bonheur d'un homme au prix du malheur des autres, accentue l'ironie: "Perderam-se uns porcos, salvou-se uma 
ama" (Evangelho: 356). L'énoncé résume le passage et concentre l'ironie, son effet renvoie à une technique ironique tirée de la théorie de l'Américain: le "ironic slap", que l'on pourrait traduire par 'gifle ironique', qui consiste à laisser place à la fin d'un paragraphe non ironique, à une phrase ironique ferme.

La perfection comme attribut du Dieu chrétien (dans la personne de Jésus) et son respect pour la vie de ses créatures sont en effet mises en doute. En outre, les "poderes milagrosos" / 'pouvoirs miraculeux' de Jésus sont qualifiés d' 'équivoques' (Evangelho: 359) par le narrateur qui mine, par ses réflexions, la plausibilité des miracles.

Le passage biblique des Evangiles qui raconte la multiplication des pains et poissons par Jésus est repris dans le roman saramaguien, donnant matière à réflexion sur la vraisemblance de faits présentés comme miraculeux dans le texte d'origine. Le narrateur du roman demande, sur un ton ironique, comment un peuple, si habitué à se déplacer et à prévoir de quoi manger, a pu se retrouver sans nourriture: "Como é que um povo tão precavido, tão acostumado a viajar e a prevenir-se de farnel mesmo quando se tratava de ir daqui além, se encountrou de repente desmunido de uma côdea de pão e de uma febra de conduto, é o que ninguém hoje consegue nem tenta explicar" (Evangelho: 360).

L'ironie du questionnement est renforcée par la mention que Jésus et ses amis avaient eux, 'au contraire des autres', prévu du poisson et du pain: "Jesus [...] seus amigos [...] ao contrário do resto da gente, tinham-se aviado com alguns peixes e alguns pães" (Evangelho: 360).

À ce récit sur le miracle de la multiplication des aliments où le narrateur semble reconnaître à Jésus la faculté d'accomplir des miracles, succède, à la fin du chapitre, celui d'un miracle mi-réussi mi-manqué. Jésus brûle un figuier sur ce seul ordre: "Nunca mais nascerá fruto de ti, e naquele mesmo instante secou a figueira", puis se repentant, lui ordonne de ressusciter: "Jesus ordenou à figueira que resuscitasse, mais ela estava morta" (Evangelho: 362), mais cette fois l'ordre reste vain car le figuier ne revit pas. La fausse conciliation ou reconnaissance apparente par le narrateur des 'pouvoirs miraculeux' du personnage, suivie d'un miracle raté en fin de chapitre, rappelle, une nouvelle fois, la technique de la gifle ironique.

Le repentir des figures liées à la divinité, est repris dans d'autres romans du corpus. Dans, História, Saint Antoine, après avoir arraché les yeux d'un hérétique, les lui restitue par compassion. D'ailleurs, les miracles attribués au saint au cours du dix-septième chapitre du roman sont aussi invraisemblables que nombreux. Le repentir de Dieu est également présent dans La valse aux adieux de Kundera, avec une citation de la Genèse: “j'exterminerai de la face de la terre l'homme que j'ai créé, car je me repens de l'avoir fait" (La valse aux adieux: 150). La présence du repentir met en question la sagesse divine et rapproche ces figures de l'humanité.

En outre, dans História, l'évocation du "milagre de Ourique" (História: 20) de l'apparition miraculeuse du Christ au roi portugais qui aboutit au triomphe de ce dernier sur les Maures, est commentée par le narrateur en ces termes: "mas Cristo não quis aparecer aos mouros, e foi pena, que em vez da crudelíssima batalha poderíamos, 
hoje [...] a conversão maravilhosa dos cento e cinquenta mil bárbaros que afinal ali perderam a vida, um desperdício de almas de bradar aos céus" (História: 20). Encore une fois, le Dieu (dans la personne du Christ) dont la perfection suppose qu'il aime toute sa création et qu'il connaît le futur, laisse massacrer les Maures. Il a favorisé un côté (celui de l'armée chrétienne portugaise) aux dépens d'un autre qu'il semble avoir ignoré.

Les actions divines mal intentionnées ou maladroites par distraction présentent Dieu comme faillible ou malveillant.

La technique du changement ou de l'élargissement de la perspective est donc créatrice d'ironie par le moyen de la parodie d'un pré-texte facilement reconnaissable. Dans Evangelho, la mention d'autres victimes innocentes sert le même but. Cette fois c'est l'action d'un ange qui est au centre de l'attention. Il s'agit de l'ange qui a annoncé à Joseph qu'il devait s'enfuir pour sauver l'enfant Jésus du massacre ordonné par Hérode. Le narrateur se demande pourquoi l'ange n'est pas aussi apparu aux autres parents et n'a pas sauvé la vie de leurs enfants.

Saramago, par un effet de contraste, rend ironique le commentaire du narrateur. Tout d'abord ce dernier feint de reconnaître les qualités des anges: "os anjos existem para tornar-nos a vida fácil, amparam-nos quando vamos a cair ao poço, guiam-nos pelo braço quando estamos quase a ser atropelados por uma quadriga sem freio ou por um automóvel sem travões..." (Evangelho: 126).

Mais une lecture plus attentive montre que ces qualités correspondent plus à celles d'un héros de bande dessinée qu'à celles, en principe très nobles, que le récit biblique attribue aux anges (annoncer le péril de mort et l'accomplissement de la volonté divine). La présentation caricaturale des anges est achevée par la banalisation de leur rôle (nous sauver de la chute dans un puits ou d'un accident de voiture). Le narrateur fait remarquer que l'omission de l'ange a coûté la vie aux enfants et démystifie ainsi l'efficacité de son rôle protecteur: "Um anjo realmente merecedor desse nome até podia ter poupado o pobre José a estas agonias, bastava que aparecesse em sonho aos pais dos meninos de Belém, dizendo a cada um, Levanta-te, toma o menino e sua mãe, foge para o Egipto e fica lá até que eu te avise [...] desta maneira salvavam-se os meninos todos..." (Evangelho: 126). L'imperfection divine est soulignée par la mention des erreurs commises par des figures (personnes) liées à Dieu: Jésus, les anges et, comme on le verra après, Dieu lui-même.

Le narrateur d'Evangelho présente une autre victime innocente de Dieu. Il fait allusion au Livre de Job de l'Ancien Testament qui fait l'objet d'une dispute entre Dieu et Satan, tous deux campés dans leurs idéologies. Le cas de Job dresse, dans le roman, l'image d'un Dieu cruel en même temps qu'il vient allonger la liste de ses victimes: "o Senhor já teria mandado castigo, sem pau nem pedra, como é seu costume, haja vista o caso de Job, arruinado, leproso, e mais sempre havia sido varão íntegro e recto, temente a Deus, a sua pouca sorte foi ter-se tornado en involuntário objecto de uma disputa entre Satanás e o mesmo Deus, cada qual agarrado às suas ideias e prerrogativas" (Evangelho: 133). 
Dans un autre roman de Saramago, Memorial, le discours du prêtre Bartolomeu, l'un des personnages principaux, expose la réflexion suivante sur le Dieu manchot: “"eu te digo que maneta é Deus, e fez o universo [...] não está escrito, só eu digo que Deus não tem a mão esquerda, porque é à sua direita, à sua mão direita, que se sentam os eleitos, não se fala nunca da mão esquerda de Deus, nem as Sagradas Escrituras, nem os Doutores da Igreja, à esquerda de Deus não se senta ninguém, é o vazio, o nada, a ausência, portanto Deus es maneta [...] da mão esquerda" (Memorial: 68).

Pour le prêtre de Memorial, le monde a été créé par un Dieu manchot, imparfait. L'inutilité de la main gauche d'un Dieu qui met à sa droite les élus, l'omission de la mention de sa main gauche dans les textes sacrés impliquent, selon le discours du prêtre, l'absence de cette main. Dieu est donc manchot, défaut qui n'est pas pourtant une limitation, car il a achevé la création, mais qui fait de lui un Dieu imparfait dans le monde romanesque saramaguien.

Que la réflexion sur l'imperfection divine se trouve dans le discours du prêtre (représentant de Dieu) est un choix chargé d'ironie. La réflexion du prêtre parodie le texte biblique (Genèse, Apocalypse) par la reprise de certains éléments: la mention de la main droite du Dieu, l'identification du côté droit comme celui des élus et l'attribution de la création universelle à Dieu. Tous servent à identifier la divinité de Memorial à celle de la Bible, rapprochement qui augmente l'effet ironique né de l'imperfection qui est imputée à cette dernière.

Le chapitre vingt-deux d'Evangelho décrit une rencontre entre Jésus, Dieu et le diable et permet d'éclaircir le projet de Dieu et de développer le parallélisme entre Dieu et le diable qui traverse le roman.

Le plan de Dieu, exposé à la première personne, montre la nature et l'ambition du personnage: "este inquieto coração meu que todos os dias me diz [...] bonito destino, arranjaste, depois de quatro mil anos de trabalho [...] continuas a ser o deus de um povo pequeníssimo que vive numa parte diminuta do mundo..." (Evangelho: 369-370).

Il n'a aucun souci de l'amour ou du bonheur humains. Il s'agit d'un projet dont il est le seul bénéficiaire. Il souhaite devenir le dieu du plus grand nombre pour étendre son pouvoir avec l'aide de Jésus: "ajudar, podes, Ajudar a quê, A alargar a minha influência, a ser deus de muito mais gente..." (Evangelho: 370 ).

Dieu est peint, dans son propre discours, comme un être égoïste. Le ton cynique qu'il adopte lui confère une personnalité égoïste et méchante, bien proche de celle du diable. Dieu accepte qu'il se serve de l'Homme en fonction de ses convenances et manifeste une conception servile ou aliénée de l'humanité: "Então, servis-vos dos homens, Sim, meu filho, o homem é pau para toda a colher, desde que nasce hasta que morre está disposto a obedecer, manda-no para ali, e ele vai, dizem-lhe que pare, e ele pára..." (Evangelho: 372).

\footnotetext{
${ }^{4}$ Cette partie du roman est, probablement, à l'origine du choix du titre de la traduction française de Memorial do Convento par Le Dieu manchot.
} 


\section{$144 \square$ LA PROFANATION DU SACRÉ DANS CERTAINS ROMANS}

L'insertion dans son discours de l'énumération par ordre alphabétique ${ }^{5}$ des morts humains nécessaires à la réalisation de son projet, achève de manière ironique le dessein d'un Dieu égoïste et cruel: "Deus suspirou e, nom tom monocórdico [...] começou a ladainha, por ordem alfabética [...] Adalberto de Praga, morto com um espantão de sete pontas, Adriano, morto à martelada sobre uma bigorna, Afra de Ausburgo, morta na fogueira, Agapito de Preneste, morto na fogueira..." (Evangelho: 381).

Au cours de l'énumération, Dieu fait allusion aux morts de Jean et Marie Madeleine par l'adjectif, souvent dépréciatif, "Esses" / 'ces...-là': "João e Maria de Magdala, Esses morrerão de sua natural morte" (Evangelho: 381). De plus, la répétition du mot "ídem" (au moins dix-huit fois) pour indiquer que tel ou tel sont morts sous une torture similaire, manifeste l'ennui de Dieu. Ces éléments exagèrent (hyperbole) le cynisme et l'indifférence divins.

L'énumération, chaotique par son extension (quatre pages), s'intègre dans le discours du responsable de ces morts. L'ensemble des données fait du chapitre vingt-deux d'Evangelho un échantillon de l'ironie saramaguienne par le biais de la parodie de la source biblique.

La critique du pouvoir de l'Eglise catholique y est également incluse car le projet de Dieu décrit dans le roman, implique que l'institution a obtenu sa puissance au prix de nombreux morts.

Le narrateur d'Evangelho réfléchit sur l'invraisemblance de certaines croyances soutenues par la foi ou les intérêts de l'Église catholique. Les réflexions sur l'authenticité et la valeur des reliques sont une source. C'est le cas du prépuce de l'enfant Jésus qui fournit matière aux commentaires ironiques du narrateur: "o prepúcio da chorosa criança, cujo destino, do prepúcio falamos, não do menino, daria por si só um romance, contado a partir deste momento, em que não passa de um pálido anel de pele que apenas sangra, e a sua santificação gloriosa, quando foi papa Pascoal I, no oitavo século desta nossa era. Quem o quiser ver, hoje, não tem mais do que ir à paróquia de Calcata [...] onde relicariamente se mostra..." (Evangelho: 89).

Le commentaire est fait sur un registre inapproprié par rapport au ton solennel qui convient à l'évocation d'une relique considérée comme sainte. En effet le ton du narrateur se rapproche de celui d'un guide touristique: 'Celui que veut voir le prépuce de Jésus aujourd'hui, il n'a qu'à se rendre à Calcata'.

Une période de temps très longue, qui débute au moment de la circoncision de l'enfant, se poursuit, par un écart de huit siècles avec celui de sa sanctification et qui s'achève au présent sert à signaler l'invraisemblance de l'authenticité de la relique. L'insertion des données qui semblent sérieuses (datation et personnage historiques) ${ }^{6}$

\footnotetext{
${ }^{5}$ Technique utilisée dans Memorial dans l'énumération des ouvriers.

${ }^{6}$ Pascal I fut pape au IX siècle et la ville de Calcata est seulement une ville parmi plusieurs autres à avoir affirmé posséder la relique (J. Mathieu-Rosay, Chronologie des papes, Belgique / France, Alleur / Marabout, 1988). On retrouve la même technique dans Memorial (: 25), où l'inclusion d'éléments propres au registre historique, comme une date (mille six cent vingt-quatre) et la mention d'un person-
} 
relève la durée et octroie réalisme et crédibilité aux informations du narrateur. La description que ce dernier fait de la relique comme un 'pâle anneau de peau', contribue à banaliser sa valeur.

Il en va de la fausseté des données comme du ton irrévérencieux du narrateur, qui créent un effet d'incongruité comique. Nous sommes face à une parodie (des registres historiques et des guides touristiques) ironique (le narrateur qui feint de donner des renseignements vrais) et comique (créé par l'incongruité entre le registre utilisé et la solennité attendue).

Nous avons signalé auparavant l'intérêt de Hutcheon de dégager la parodie du comique, mais l'exemple précédent est contraire, ce qui nous permet de parler de Margaret Rose qui, dans Parody: ancient, modern, and post-modern (1993), conteste l'exclusion par Hutcheon, de la forme comique de sa définition de la parodie (Rose: 228) qu'elle voit comme le résultat de la volonté de différencier la parodie du burlesque.

Pour Rose la parodie peut être en même temps comique et métafictionnelle, une combinaison plus complexe de ces deux critères et des formes reliées. Celle-ci interagit de manière spéciale avec l'ironie dans la littérature contemporaine où 1 'ironie stable' est moins présente, notion que Booth définissait en 1974 comme celle dont le lecteur peut identifier, de façon précise, l'intention sous-jacente.

Le registre utilisé par Saramago dans l'exemple ultérieur, inapproprié pour le traitement d'un sujet "sacré", signale ici la présence de l'ironie dans un discours qui cherche à montrer l'invraisemblance de ce qu'il présente comme vrai.

Les saints font partie des éléments empruntés au récit religieux dans les romans du corpus. Dans Memorial de Saramago, la nature divine ou supra humaine du miracle ainsi que sa véracité certifiée ("veros e certificados milagres") sont discréditées par les commentaires du narrateur.

Le deuxième chapitre du roman s'ouvre sur l'évocation d'un événement jugé miraculeux "o nascimento do infante" (Memorial: 19), la naissance de l'héritier de la couronne, l'origine divine la grossesse de la reine "obséquio divino". Viennent ensuite trois miracles liés à l'ordre franciscain. ${ }^{7}$ Le narrateur fait remarquer, sur un ton moqueur, que le dernier de ces miracles s'est passé malgré la présence de Saint Antoine, dont la chapelle était à côté: "Santo António que, tendo cometido até hoje tantos e tão variados milagres, também podia ter feito este" (Memorial: 25).

L'ironie est créée par le contraste entre le rappel de "tantos e tão variados milagres" I 'grand nombre et variété des miracles' accomplis par le saint et le fait que le vol s'est passé malgré sa présence. Le recueil des miracles finit sur une affirmation: "Santo António o mais milagroso dos santos, mormente para encontrar coisas perdidas" (Memorial: 25)

nage historique (le roi Philippe), donne aux fausses informations du narrateur un caractère authentique et vérifiable qui contraste avec l'invraisemblance des miracles.

${ }^{7}$ Celui d'un moine dont le corps ne s'est pas corrompu trois jours après la mort, celui du repentir d'un voleur qui retrouve la santé grâce à la transpiration d'un saint en bois et finalement la récupération des lampes qui avaient été volées dans un couvent franciscain. 
/ 'San Antonio, le plus miraculeuse des saints notamment pour retrouver ce qui a été perdu'. Mais aucun des miracles cités ne sert à justifier cette idée, au contraire, le dernier la contredit puisque les lampes volées en dépit de la présence du Saint ont été retrouvées grâce à un fidèle. L'affirmation citée auparavant devient de ce fait ironique.

Quant à la nature divine du miracle de la naissance de l'héritier de la couronne, elle est mise en question puisqu'elle est décrite comme une chose que l'on prépare:

"Bem servido de milagres, igualmente. Ainda é cedo para falar deste que se prepara" (Memorial: 19). Le narrateur attribue la préparation du miracle à l'ordre franciscain, présenté au premier chapitre comme porteur d'une certitude qui lui permet de négocier avec le roi: si le roi construit un couvent franciscain, Dieu lui donnera un héritier / "que se vossa majestade prometesse levantar um convento na vila de Mafra, Deus lhe daria sucessão" (Memorial: 14). Le mot préparer montre que la certitude de la grossesse de la reine n'est pas d'origine "divine" mais humaine.

La naissance de l'héritier perd le 'statut' de miracle dans la clôture du deuxième chapitre où le narrateur, sur un ton ironique marqué par le désir apparent de dissiper des malentendus, signale que la certitude des Franciscains tire son origine de la divulgation d'un secret entendu en confession: "Agora não se vá dizer que, por segredos de confissão divulgados, souberam os arrábidos que a rainha estava grávida antes mesmo que ela o participasse ao rei" (Memorial: 26).

L'utilisation de la négation ("não se vá dizer que") éclaircit ironiquement le lecteur sur la manipulation à leur profit de l'information par les Franciscains, constat que le narrateur amène le lecteur à tirer par la mention du projet, depuis longtemps sollicité par l'ordre, de la construction d'un couvent: "convento em Mafra o anda a querer a ordem de S. Francisco desde mil seiscentos e vinte e quatro..." (Memorial: 25).

Ce deuxième chapitre de Memorial, procède, dès les premières pages, à la démystification des miracles. Son contenu constitue un paramètre pour l'analyse de nouvelles allusions aux miracles dans les chapitres suivants du même roman et, de manière intertextuelle, des romans postérieurs. En effet, comme nous l'avons vu auparavant, les miracles de Saint Antoine sont mis en question dans História (au dix-septième chapitre) et dans Evangelho il est question de ceux de Jésus.

Cette réflexion sur la profanation du sacré, met en évidence les différentes manières avec lesquelles deux romanciers contemporains créent l'ironie en parodiant l'intertexte biblique.

Nous avons constaté que la parodie biblique est un point de convergence des écritures romanesques des deux écrivains. En effet, l'intertexte biblique est une des sources communes des romans du corpus. Toutefois des particularités stylistiques se dessinent.

Chez Saramago sont parodiées des figures bibliques comme Jésus (Evangelho) ou le Bon Samaritain (Ensaio). La mention de ces figures est directe si l'on pense à la parodie biblique dans l'œuvre de Kundera plus allusive et ambiguë. Mais autant Saramago que Kundera mentionnent, parfois, dans les commentaires métafictionnels du narrateur, l'intertexte qu' ils parodient (comme celui du livre de Job par le narrateur d'Evangelho ou celui de la Genèse dans La vie est ailleurs). 
L'analyse montre que l'ironie naît du décalage entre les figures parodiées dans les romans du Portugais par rapport au texte source. Ainsi le Jésus d'Evangelho n'accomplit pas le miracle de la résurrection de Lazare. En revanche il accomplit des miracles incertains qui font des victimes innocentes. Quant au personnage identifié au Bon Samaritain dans Ensaio, il est un voleur qui profite de l'aveuglement de sa victime, action incongrue qui génère de l'ironie.

La grande ressemblance entre les personnages des romans de Saramago et les figures de l'intertexte biblique sert d'arrière plan au contraste ironique. Le lecteur, tout au moins habitué à les accepter tels qu'ils sont exposés dans la Bible, les retrouve cités dans le roman où il est confronté au mécompte de ses attentes.

Quant aux romans de Kundera, la parodie biblique passe par l'adoption d'un ton et un lexique qui renvoient à cette source: “Au commencement, il n'y avait que lui, Jaromil", dit le narrateur de La vie est ailleurs. Le récit est apparemment étranger à celui de la Bible, jusqu'au moment où, dans le lexique, figurent les mots "création", "homme", "femme", "Dieu". Seule la relecture rend possible l'association de ces éléments et la compréhension de la parodie biblique et sa possible signification. Les éléments du texte parodié sont placés dans un tout autre contexte, établissant ainsi un décalage par rapport au texte source et qui, par un effet de défamiliarisation, peut donner lieu à l'ironie et au rire.

Nous avons vu aussi comment la parodie ironique faite par Kundera travaille dans deux directions: elle accentue et détruit. Ainsi, dans La vie est ailleurs, l'écrivain transpose-t-il le passage biblique de la trahison de Pierre dans une scène relatant la lâcheté de Jaromil. L'écart entre la nature des faits mis en rapport: l'apôtre reniant Dieu, Jaromil, sa petite amie, accentue le dédain chez Jaromil, en même temps qu'il banalise celui de l'apôtre, donnant lieu à une ironie caustique.

Nous résumons la vision de Hutcheon pour qui la parodie moderne est une parodie ironique, dans la mesure où elle achève un contraste ironique entre le texte parodié et le texte parodique. Elle est aussi "une forme de critique littéraire" (le parodiste fait une évaluation en choisissant le texte à parodier) immanente, métalittéraire (elle est dans le texte) et synthétique (plus qu'analytique), en plus qu'un "instrument de libération" utilisé par le parodiste pour "exorciser ses fantômes personnels" ("Ironie et": 474) ou de les utiliser à des fins qui lui conviennent.

De ce fait, étudier la parodie biblique permet efficacement de saisir des nombreuses techniques utilisées dans le roman contemporain: l'énumération chaotique ou non, le choix de virgules au lieu des points, la répétition, la transposition du texte parodié dans un nouveau contexte qui banalise ou change sa signification originale, l'appropriation du lexique, ton et figures du texte parodié.

L'Ancien et le Nouveau Testament sont tout autant parodiés dans les romans du corpus, notamment les livres de la Genèse, Job, l'Exode, les Evangiles et l'Apocalypse.

Dans la partie sur l'ironie de la divinité perfectible, nous avons constaté sa prédominance dans l'œuvre de Saramago, thème où, dans son écriture romanesque, la création ironique prend tout son sens. L'imperfectibilité de Dieu, qui est évoquée dans Levantado 
do Chão, Evangelho, História et Memorial, donne lieu à l'ironie par des techniques variées: la méditation sur l'invraisemblance des miracles, l'attribution de conduites blâmables aux représentants religieux, l'utilisation de registres inappropriés dans le traitement du sacré, la répétition (notamment celle qui rappelle que les premiers à avoir identifié Jésus comme "fils de Dieu" furent les démons), la présentation des nouvelles perspectives des valeurs dogmatiques et des figures religieuses. Ces techniques généralement sont placées dans les commentaires du narrateur.

Dans ces romans de Saramago, il est question des prêtres hypocrites (Levantado do Chão), d'un dieu manchot (Memorial) et d'un Jésus qui se repent, commet des erreurs et semble plus proche de l'humanité que de la divinité (Evangelho).

Malgré la prédominance du thème de l'imperfectibilité de Dieu dans les romans de Saramago comme source d'ironie, il est également présent chez Kundera où l'imperfectibilité de la divinité passe, comme chez Saramago, par l'attribution de conduites blâmables aux représentants religieux ou très décalées par rapport à leur désignation, mais aussi en donnant à ses personnages les qualificatifs de "prédicateurs" et d' “anges", comme c'est le cas pour Bertlef dans La valse aux adieux. Autre convergence entre Kundera et le Nobel portugais pour la création de l'ironie, est celle de représenter une divinité qui se repent. Cependant à la différence que, chez l'auteur franco-tchèque, le repentir de Dieu concerne la création humaine (La valse aux adieux).

Nous avons pu constater, à travers l'analyse de l'ironie et la parodie dans les romans des deux auteurs, leurs points de convergence et leurs particularités stylistiques. Dans cette étude ont été mises en exergue des notions comme "tranchant ironique" (Hutcheon), "ironie stable" et "gifle ironique" (Booth).

L'approche théorique nous fait comprendre pourquoi la définition de l'ironie comme "antiphrase" est inadéquate pour analyser les énoncés ironiques des romans du corpus. En effet, elle est une stratégie qui surpasse le statut de trope ou figure rhétorique pour devenir une forme structurante du roman et une stratégie intertextuelle. La présence de l'ironie dans ces romans est souvent aux côtés du genre parodique avec lequel elle interagit.

\section{Oeuvres citées}

Booth, Wayne C. 1974. A Rhetoric of Irony. Chicago / London: University of Chicago.

HAMON, Philippe. 2002. L'ironie littéraire. Essai sur les formes de l'écriture oblique. París: Hachette.

HutCHeOn, Linda. 1994. Irony's edge. London / New York: Routledge. 1993. "La política de la parodia postmoderna". Criterios. Teoría de la literatura y de las artes, estética, cultorología. México: UAM-Xochimilco, Juillet, pp. 187-201. Trad. de "The Politics of Postmodern Parody". Heinrich F. PLETT, ed., 1991. Intertextuality. Berlin / New York: Walter de Gruyter. Pp. 225-236. 
. 1985. A Theory of Parody. The Teachings of Twentieth Century Art Forms. New York / London: Methuen.

.1981. "Ironie, satire et parodie. Une approche pragmatique de l'ironie". Poétique, $\mathrm{N}^{\circ}$ 46. Paris: Le Seuil. Pp. 140-155.

.1978. "Ironie et parodie: stratégie et structure". Poétique, № 36. Paris: Le Seuil. Pp. 467-477.

KUnDERA, Milan. 2003. La valse aux adieux. Paris: Gallimard. .2003. La vie est ailleurs. Paris: Gallimard.

Mathieu-Rosay, J. 1988. Chronologie des papes. [Belgique] / [France]: Alleur / Marabout.

RoSE, Margaret A. 1993. Parody: ancient, modern, and post-modern. Cambridge: University of Cambridge.

SARAMAGO, José. 1995. Ensaio sobre a Cegueira. Lisboa: Caminho. . 1991. O Evangelho segundo Jesus Cristo. Lisboa: Caminho. . 1989. História do Cerco de Lisboa. Lisboa: Caminho. 1987. Memorial do Convento. Lisboa: Caminho. 1986. Levantado do Chão. Lisboa: Caminho.

ZAVALA, Lauro. 1996. "Glosario para el estudio de la ironía literaria". Sincronía. México: UAM- Xochimilco. Hiver. En ligne. http://sincronia.cucsh.udg.mx/zavalo.html. 